\title{
About Two-dimensional Mathematical Model of Contaminant Migration in Unsaturated Catalytic Porous Media with Traps
}

https://doi.org/10.31713/MCIT.2020.35

\author{
Anatolyy Vlasyuk \\ Department of Economics, Mathematical \\ Modeling and Information Technologies \\ The National University of Ostroh \\ Academy \\ Ostroh, Ukraine \\ Anatoliy.Vlasyuk@oa.edu.ua
}

\author{
Viktor Zhukovskyy, Natalya Zhukovska \\ Department of Applied Mathematics \\ National University of Water and \\ Environmental Engineering Rivne, Ukraine \\ V.V.Zhukovskyy@nuwm.edu.ua, \\ N.A.Zhukovska@nuwm.edu.ua
}

\author{
Serhiy Kraychuk \\ Department of Economic \\ Cybernetics \\ Rivne State University of \\ Humanities \\ Rivne, Ukraine \\ Laxxik@gmail.com
}

\begin{abstract}
This paper proposes an approach for the computer simulation of complex physical problem of contaminant migration through unsaturated catalytic porous media to the filter-trap. The corresponding mathematical model in the two-dimensional nonlinear case is presented. The model includes detailed considerations of the moisture transfer of saline solutions under the generalized Darcy's and Cluta's laws in different subregions of soil. The numerical solution of the boundary value problem was found by the finite difference method and proposed the algorithm for computer implementation. The proposed algorithm may be used for creating software with effective risk assessment strategies and predicting the cleaning and further useful use of the soil massifs.
\end{abstract}

Keywords-mathematical model; computer modelling; moisture transfer; boundary-value problem; numerical method; refinement; NanoSurface

\section{INTRODUCTION}

Contaminant sources occur in soil, from which the chemicals then migrate to air, surface water, and groundwater [1]. Predicting the movement of contaminants through porous media requires addressing the fate and transport processes that predominate in each sub medium and integrating the interactions between the media. This is a quite complicated problem [2-6].

In previous works, we investigated similar physical problems for saturated media [7, 8]. Therefore, in the zone of suspended water different processes became commensurate (e.g. the convective term and diffusion are almost identical due to the low velocity of moisture transfer). This fact opens a widespread use of colloid adsorbents in purification processes $[9,10]$. Thus, the next mathematical model is presented for the first time and take into account a wide range of factors.

\section{FORMULATION OF THE PROBLEM}

The filter-trap located at a depth $C D$ of the soil. The pore spaces between the soil grain particles are partially filled with water and partially with air (zone of suspended water). There are dozens of colloid adsorbents with radius $R$ (micro or nanoparticles) in the layer of soil. Therefore, they may be used in for cleaning purposes as well. There is a piezometric pressure on the upper and filter-trap surfaces $\tilde{H}_{1}$ and $\tilde{H}_{2}\left(\tilde{H}_{1}>\tilde{H}_{2}\right)$, respectively. The contaminants concentrations at the initial time $t=0: \tilde{C}_{1}^{0}(x, y)$ (for a saline solution in soil pore network, $>300 \mu \mathrm{m}[11]$ ), $\tilde{C}_{2}^{0}(x, y)$ (soil surfaces hold water in soils and prevent rapid movement in smaller pores, thus the contaminant are held on the surface of the ground skeleton), $\tilde{C}_{3}^{0}(x, y)$ (for contaminant located in the soil skeleton, the importance of such factor was presented in [12]), and $\tilde{Q}^{0}(x, y, r)$ (for contaminant inside microparticles with radius $R$ [13]) are known.

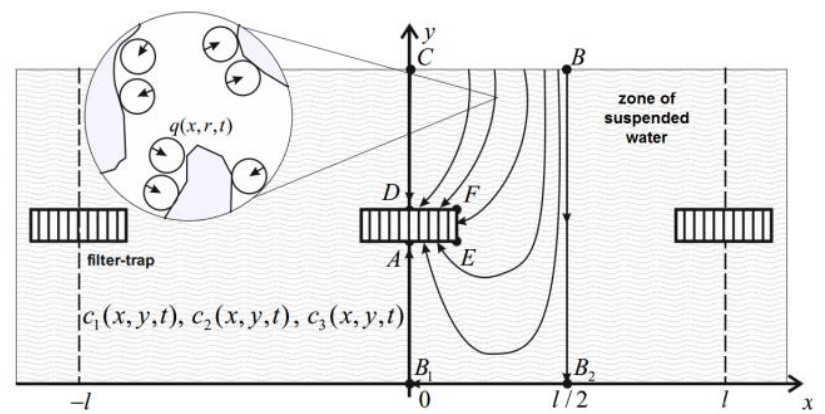

Figure 1. The process of contaminant migration in unsaturated twodimensional catalytic porous media to the filter-trap 


\section{Modeling, control and information technologies $-\mathbf{2 0 2 0}$}

The soil concentrations $\tilde{C}_{1}^{1}(t), \tilde{C}_{2}^{1}(t)$ and $\tilde{C}_{3}^{1}(t)$ on the upper surface and $\tilde{C}_{1}^{2}(t), \tilde{C}_{2}^{2}(t), \tilde{C}_{3}^{2}(t)$ for the filter-traps are also known.

It is necessary to find out the $c_{1}(x, y, t), c_{2}(x, y, t)$, $c_{3}(x, y, t)$ and $q(x, y, r, t)$ concentrations distribution over the soil.

\section{MATHEMATICAL MODEL}

Due to the symmetry of the filtration picture a fragment of $\mathrm{AB}_{1} \mathrm{~B}_{2} \mathrm{BCD}$ filtration area is considered. Therefore, the boundary value problem of the contaminant transport in a porous medium in a two-dimensional nonlinear case was solved using a mathematical model with the following equations $[7$, $14,15]$

$$
\begin{aligned}
& \frac{\partial\left(D_{1}\left(c_{1}\right) \frac{\partial c_{1}}{\partial x}\right)}{\partial x}+\frac{\partial\left(D_{1}\left(c_{1}\right) \frac{\partial c_{1}}{\partial y}\right)}{\partial y}- \\
& -v_{x} \frac{\partial c_{1}}{\partial x}-v_{y} \frac{\partial c_{1}}{\partial y}-\gamma_{1} c_{1}+\gamma_{2} c_{2}=\frac{\partial\left(\theta c_{1}\right)}{\partial t}, \\
& \frac{\partial\left(D_{2}\left(c_{2}\right) \frac{\partial c_{2}}{\partial x}\right)}{\partial x}+\frac{\partial\left(D_{2}\left(c_{2}\right) \frac{\partial c_{2}}{\partial y}\right)}{\partial y}+ \\
& +\gamma_{1} c_{1}-\gamma_{2} c_{2}+\gamma_{3} c_{3}-\left.\theta_{0} \frac{\partial q}{\partial r}\right|_{r=R}=\frac{\partial c_{2}}{\partial t}, \\
& \frac{\partial\left(D_{3}\left(c_{3}\right) \frac{\partial c_{3}}{\partial x}\right)}{\partial x}+\frac{\partial\left(D_{3}\left(c_{3}\right) \frac{\partial c_{3}}{\partial y}\right)}{\partial y}+ \\
& +\gamma_{2} c_{2}-\gamma_{3} c_{3}=\frac{\partial c_{3}}{\partial t} \\
& \mu(h) \frac{\partial h}{\partial t}=\frac{\partial}{\partial x}\left(K\left(h, c_{1}\right) \frac{\partial h}{\partial x}\right)+\frac{\partial}{\partial y}\left(K\left(h, c_{1}\right) \frac{\partial h}{\partial y}\right)- \\
& -\frac{\partial}{\partial x}\left(v_{c} \frac{\partial c_{1}}{\partial x}\right)-\frac{\partial}{\partial y}\left(v_{c} \frac{\partial c_{1}}{\partial y}\right)+f, \\
& \frac{1}{r^{2}} \frac{\partial}{\partial r}\left(r^{2} D_{0}(q) \frac{\partial q}{\partial r}\right)=\frac{\partial q}{\partial t} \\
& \mathrm{v}_{x}=-K\left(h, c_{1}\right) \frac{\partial h}{\partial x}+v \frac{\partial c_{1}}{\partial x}, v_{y}=-K\left(h, c_{1}\right) \frac{\partial h}{\partial y}+v \frac{\partial c_{1}}{\partial y} \text {, } \\
& \frac{\partial v_{x}}{\partial x}+\frac{\partial v_{y}}{\partial y}=0 \text {, } \\
& \left.q(x, r, t)\right|_{r=R}=\frac{k_{f} \cdot c_{2}^{\beta}(x, t)}{1+\eta \cdot c_{2}^{\beta}(x, t)}, \\
& \left.\frac{\partial h}{\partial n}\right|_{A B_{1} \cup B_{1} B_{2} \cup B_{2} B \cup C D}=0,\left.h\right|_{C B}=H_{1},\left.h\right|_{A E \cup E F \cup F D}=H_{2} \text {, } \\
& \left.l_{1} c_{1}\right|_{C B}=\tilde{C}_{1}^{1}(t),\left.l_{2} c_{2}\right|_{C B}=\tilde{C}_{2}^{1}(t),\left.l_{3} c_{3}\right|_{C B}=\tilde{C}_{3}^{1}(t),
\end{aligned}
$$

$$
\begin{gathered}
\left.\frac{\partial c_{1}}{\partial n}\right|_{\Gamma}=\left.\frac{\partial c_{2}}{\partial n}\right|_{\Gamma}=\left.\frac{\partial c_{3}}{\partial n}\right|_{\Gamma}=0, \\
\Gamma=B B_{2} \cup B_{2} B_{1} \cup B_{1} A \cup A E \cup E F \cup F D \cup D C, \\
\left.c_{1}\right|_{t=0}=\tilde{C}_{1}^{0}(x, y),\left.c_{2}\right|_{t=0}=\tilde{C}_{2}^{0}(x, y), \\
\left.c_{3}\right|_{t=0}=\tilde{C}_{3}^{0}(x, y), \\
\left.q\right|_{t=0}=\tilde{Q}^{0}(x, y, r),\left.\frac{\partial q(x, y, r, t)}{\partial r}\right|_{r=0}=0,
\end{gathered}
$$

where $c_{1}(x, y, t), D_{1}$ - concentration and convective diffusion coefficient of contaminant in filtration flow; $c_{2}(x, y, t), D_{2}-$ concentration and molecular diffusion coefficient of contaminant in water connected with soil skeleton; $c_{3}(x, y, t)$, $D_{3}$ - concentration and diffusion coefficient of contaminant in soil skeleton; $q(x, y, r, t), \quad D_{0}$ - concentration and diffusion coefficient of contaminant in particles with radius $R$, which in soil skeleton; $k_{f}, \beta, \eta$-adsorption isotherm coefficients; $\theta_{0}-$ coefficient of micro- or nanoparticle mass transfer influence on mass transfer near the ground skeleton; $\vec{v}=\left\{v_{x}, v_{y}\right\}$ - filtration velocity; $K$ - moisture expansion coefficient; $\gamma_{1}, \gamma_{2}, \gamma_{3}$ mass transfer coefficients; $(x, y) \in \Omega$-coordinates; $l_{i}, i=\overline{1,3}$ - differential operators for boundary conditions; $t$ - time, $0<t<t_{1}, r$ - radius (radial variable) $0<r<R$.

The equations above describe the following transport mechanisms of contaminant with concentration: (1) $c_{1}$ in a convectively mobile pore solution; (2) $c_{2}$ in the water bound with the soil skeleton tacking into account the intra-particle diffusion; (3) $c_{3}$ in the soil skeleton. Equation (4) describes moisture transfer; (5) the intra-particle transport mechanisms of contaminant with concentration $q[13,16] ;$; 6 ) the generalized equation of the Darcy-Cluta law in the two-dimensional case for moisture transfer of the salt solutions [17]; (7) the adsorption isotherm; (8) boundary conditions for piezometric head $h(x, t)$; (9)-(12) boundary conditions for concentrations $c_{1}(x, t), c_{2}(x, t)$ and $c_{3}(x, t) ;(13)$ boundary conditions for intra-particle concentrations $q(x, y, r)$.

Transfer of contaminant dissolved in water (saline solution) by filtration flow occurs under the influence of the pressure gradients and the concentration of salts. The moisture and mass transfer of saline solutions occurs under the generalized Darcy's and Cluta's laws.

The boundary value problem (1)-(13) is set correctly (or correctly posed), because the conditions of existence and uniqueness of its solution are fulfilled [18].

\section{Numerical SOLUTION OF BOUNDARY VALUE PROBLEM}

The computational mesh $\omega_{\mathrm{h} 11, \mathrm{~h} 12, \mathrm{~h} 2, \tau} \tau$, was build for finitedifference approximation with steps $\mathrm{h}_{11}, \mathrm{~h}_{12}, \mathrm{~h}_{2}$ and $\tau$ by $O x$ axis, $O y$-axis, $O r$-axis and $O t$-axis for $x, y, r, t$ variables respectively [19-21] 
Modeling, control and information technologies -2020

$$
\omega_{h_{1} h_{12} h_{2} \tau}=\left\{\begin{array}{c}
x_{i_{1}}=i_{1} h_{11}, y_{i_{2}}=i_{2} h_{12}, r_{j}=j h_{2}, t_{k}=k \tau, \\
i_{1}=\overline{0, n_{11}}, i_{2}=\overline{0, n_{12}}, j=\overline{0, n_{2}}, k=\overline{0, n_{3}}, \\
h_{11} n_{11}=l_{1}, h_{12} n_{12}=l_{2}, h_{2} n_{2}=R, \tau n_{3}=T,
\end{array}\right\}
$$

where $n_{11}, n_{12}, n_{2}, n_{3}-$ steps count.

Let us show the solution algorithm on the example of an equation (2). According to a locally one-dimensional method, we represent the differential equation (2) as a system of two one-dimensional equations with locally one-dimensional method [14]:

$$
\begin{aligned}
& D_{2} \frac{\partial^{2} c_{2}}{\partial x^{2}}+\frac{\gamma_{1} c_{1}}{2}-\frac{\gamma_{2} c_{2}}{2}+\frac{\gamma_{3} c_{3}}{2}-\left.\frac{\theta}{2} \frac{\partial q}{\partial r}\right|_{r=R}=\frac{1}{2} \frac{\partial c_{2}}{\partial t} \\
& D_{2} \frac{\partial^{2} c_{2}}{\partial y^{2}}+\frac{\gamma_{1} c_{1}}{2}-\frac{\gamma_{2} c_{2}}{2}+\frac{\gamma_{3} c_{3}}{2}-\left.\frac{\theta}{2} \frac{\partial q}{\partial r}\right|_{r=R}=\frac{1}{2} \frac{\partial c_{2}}{\partial t}
\end{aligned}
$$

where $(x, y) \in \Omega, r \in(0, R), t>0$.

Finite-difference analogues of equations (14), (15) are tacking the following form:

$$
\begin{gathered}
\frac{c_{2, i_{1}, i_{2}}^{(k+0,5)}-c_{2, i_{1}, i_{2}}^{(k)}}{\tau}=D_{2} \frac{c_{2, i_{1}+1, i_{2}}^{(k+0,5)}-2 c_{2, i_{1}, i_{2}}^{(k+0)}+c_{2, i_{1}-1, i_{2}}^{(k+0,5)}}{h_{11}^{2}}+ \\
\left.+\frac{\gamma_{1} c_{1, i_{1}, i_{2}}^{(k)}-\frac{\gamma_{2} c_{2, i_{1}, i_{2}}^{(k+0)}}{2}+\frac{\gamma_{3} c_{3, i_{1}, i_{2}}^{(k)}}{2}-}{2}\right) \\
-\frac{\theta}{2}\left(\frac{\frac{3}{2} q_{n_{2}}^{(k+0,5)}-2 q_{n_{2}-1}^{(k+0,5)}+\frac{1}{2} q_{n_{2}-2}^{(k+0,5)}}{h_{2}}\right), \\
\frac{c_{2, i_{1}, i_{2}}^{(k+1)}-c_{2, i_{1}, i_{2}}^{(k+0,5)}}{\tau}=D_{2} \frac{c_{2, i_{1}, i_{2}+1}^{(k+1)}-2 c_{2, i_{1}, i_{2}}^{(k+1)}+c_{2, i_{1}, i_{2}-1}^{(k+1)}}{h_{12}^{2}}+ \\
\left.\quad \frac{\gamma_{1} c_{1, i_{1}, i_{2}}^{(k+0,5)}}{2}-\frac{\gamma_{2} c_{2, i_{1}, i_{2}}^{(k+1)}+\frac{\gamma_{3} c_{3, i_{1}, i_{2}}^{(k+1)}}{2}-}{2}\right) \\
-\frac{\theta}{2}\left(\frac{\frac{3}{2} q_{n_{2}}^{(k+1)}-2 q_{n_{2}-1}^{(k+1)}+\frac{1}{2} q_{n_{2}-2}^{(k+1)}}{h_{2}}\right)
\end{gathered}
$$

$i_{1}=\overline{1, n_{11}-1}, i_{2}=\overline{1, n_{12}-1}, k=\overline{0, n_{3}}$.

To find the solution (16) by the Thomas method we present it in following general way:

$$
\begin{aligned}
& \left\{\begin{array}{l}
a_{i_{1}}^{2} c_{2, i_{1}-1, i_{2}}^{(k+0,5)}-\bar{c}_{i_{1}}^{2} c_{2, i_{1}, i_{2}}^{(k+0)}+b_{i_{1}}^{2} c_{2, i_{1}+1, i_{2}}^{(k+0,5)}=-f_{i_{1}, i_{2}}^{2,(k+0,5)}, \\
c_{2,0, i_{2}}^{(k+0,5)}=\mu_{11}^{2} c_{2,1, i_{2}}^{(k+0,5)}+\mu_{12}^{2}, \\
c_{2, n_{1}, i_{2}}^{(k+0,5)}=\mu_{13}^{2} c_{2, n_{11}-1, i_{2}}^{(k+0,5}+\mu_{14}^{2},
\end{array}\right. \text { where } \\
& a_{i_{1}}^{2}=\frac{D_{2}}{h_{11}^{2}}, b_{i_{1}}^{2}=\frac{D_{2}}{h_{11}^{2}}, \bar{c}_{i_{1}}^{2}=\frac{2 D_{2}}{h_{11}^{2}}+\frac{1}{\tau}+\frac{\gamma_{2}}{2},
\end{aligned}
$$

$$
\begin{gathered}
f_{i_{1}, i_{2}}^{2,(k+0,5)}=\frac{c_{2, i_{1}, i_{2}}^{(k)}}{\tau}+\frac{\gamma_{1} c_{1, i_{1}, i_{2}}^{(k)}}{2}+\frac{\gamma_{3} c_{3, i_{1}, i_{2}}^{(k+0,5)}}{2}- \\
-\frac{\theta}{2}\left(\frac{\frac{3}{2} q_{n_{2}}^{(k+0,5)}-2 q_{n_{2}-1}^{(k+0,5)}+\frac{1}{2} q_{n_{2}-2}^{(k+0,5)}}{h_{2}}\right), \\
\mu_{11}^{2}=1, \mu_{12}^{2}=0, \mu_{13}^{2}=1, \mu_{14}^{2}=0 .
\end{gathered}
$$

The stability conditions of the Thomas method are fulfilled $\left|\bar{c}_{i_{1}}^{2}\right|>\left|a_{i_{1}}^{2}\right|+\left|b_{i_{1}}^{2}\right|$. Therefore, we may find the value of concentration $c_{2}(x, y, t)$ at the each time step $(k+0.5)$ :

$$
\begin{gathered}
c_{2, i_{1}, i_{2}}^{(k+0)}=\alpha_{i_{i_{1}+1}}^{2} c_{2, i_{1}+1, i_{2}}^{(k+0,5)}+\beta_{i_{1}+1}^{2}, \text { where } \alpha_{i_{1}+1}^{2}=\frac{b_{i_{1}}^{2}}{\bar{c}_{i_{1}}^{2}-\alpha_{i_{1}}^{2} a_{i_{1}}^{2}}, \\
\beta_{i_{1}+1}^{2}=\frac{a_{i_{1}}^{2} \beta_{i_{1}}^{2}+f_{i_{1}, i_{2}}^{2,(k+0,5)}}{\bar{c}_{i_{1}}^{2}-\alpha_{i_{1}}^{2} a_{i_{1}}^{2}}, i_{1}=\overline{1, n_{11}-1}, i_{2}=\overline{1, n_{12}-1}, k=\overline{1, n_{3}}, \\
\alpha_{i_{1}}^{2}=\mu_{11}^{2}=1, \beta_{i_{1}}^{2}=\mu_{12}^{2}=0 .
\end{gathered}
$$

Correspondingly we may find the solution for (17):

$$
\begin{aligned}
& \left\{\begin{array}{l}
a_{i_{2}}^{2} c_{2, i_{1}, i_{2}-1}^{(k+1)}-\bar{c}_{i_{2}}^{2} c_{2, i_{1}, i_{2}}^{(k+1)}+b_{i_{2}}^{2} c_{2, i_{1}, i_{2}+1}^{(k+1)}=-f_{i_{1}, i_{2}}^{2,(k+1)}, \\
c_{2, i_{1}, 0}^{(k+1)}=\mu_{21}^{2} c_{2, i_{1}, 1}^{(k+1)}+\mu_{22}^{2}, \\
c_{2, i_{1}, n_{12}}^{(k+1)}=\mu_{23}^{2} c_{2, i_{1}, n_{12}-1}^{(k+1)}+\mu_{24}^{2},
\end{array}\right. \text { where } \\
& a_{i_{2}}^{2}=\frac{D_{2}}{h_{12}^{2}}, b_{i_{2}}^{2}=\frac{D_{2}}{h_{12}^{2}}, \bar{i}_{i_{2}}^{2}=\frac{2 D_{2}}{h_{12}^{2}}+\frac{1}{\tau}+\frac{\gamma_{2}}{2}, \\
& f_{i_{1}, i_{2}}^{2,(k+1)}=\frac{c_{2, i_{1}, i_{2}}^{(k+0,5)}}{\tau}+\frac{\gamma_{1} c_{1, i_{1}, i_{2}}^{(k+0,5)}}{2}+\frac{\gamma_{3} c_{3, i_{1}, i_{2}}^{(k+1)}}{2}- \\
& -\frac{\theta}{2}\left(\frac{\frac{3}{2} q_{n_{2}}^{(k+1)}-2 q_{n_{2}-1}^{(k+1)}+\frac{1}{2} q_{n_{2}-2}^{(k+1)}}{h_{2}}\right), \\
& \mu_{21}^{2}=1, \mu_{22}^{2}=0, \mu_{23}^{2}=1, \mu_{24}^{2}=1 .
\end{aligned}
$$

The concentration value $c_{2}(x, y, t)$ at the time steps $(k+1)$ is calculated using the next ratio: $c_{2, i_{1}, i_{2}}^{(k+1)}=\alpha_{i_{2}+1}^{2} c_{2, i_{1}, i_{2}+1}^{(k+1)}+\beta_{i_{2}+1}^{2}$, where $\alpha_{i_{2}+1}^{2}=\frac{b_{i_{2}}^{2}}{\bar{c}_{i_{2}}^{2}-\alpha_{i_{2}}^{2} a_{i_{2}}^{2}}, \beta_{i_{2}+1}^{2}=\frac{a_{i_{2}}^{2} \beta_{i_{2}}^{2}+f_{i_{1}, i_{2}}^{2,(k+1)}}{\bar{c}_{i_{2}}^{2}-\alpha_{i_{2}}^{2} a_{i_{2}}^{2}}, i_{1}=\overline{1, n_{11}-1}$, $i_{2}=\overline{1, n_{12}-1}, k=\overline{1, n_{3}}, \alpha_{i_{2}}^{2}=\mu_{21}^{2}=0, \beta_{i_{2}}^{2}=\mu_{22}^{2}=\tilde{C}_{2}$.

Let us write the next finite-difference equations for the initial condition as well as for boundary condition for $c_{2}(x, y, t)$ :

$c_{2, i_{1}, i_{2}}^{(0)}=\tilde{C}_{2}^{0}\left(i_{1} h_{11}, i_{2} h_{12}\right), \quad c_{2, i_{1}, n_{12}}^{(k)}=\tilde{C}_{2}^{1}\left(i_{1} h_{11}, k \tau\right), i_{1}=\overline{1, n_{11}-1}$, $k=\overline{0, n_{3}}$. 


\section{Modeling, control and information technologies -2020}

Thus, the all necessary mathematical manipulations were performed for programming language implementation of the Thomas algorithm for $c_{2}(x, y, t)$.

Similarly, we split the sub-boundary-value problems (1), (3) , (4), (5) and (6) with corresponding boundary and initial conditions (7)-(12) into the systems of one-dimensional equations. Mathematical conversions for similar onedimensional boundary-value problems are described in previous works [7, 22]. In computation algorithm we need to find out the piezometric head distribution $h(x, y, t)$ first, then the DarcyClute moisture velocity $\mathrm{v}(x, y, t)$ and finally the distribution of concentrations $c_{1}(x, t), c_{2}(x, t), c_{3}(x, t)$ and $q(x, r, t)$. Each Thomas algorithm calculations we perform at half time step $(k+0.5)$ at $O x$-axis and then at time step $(k+1)$ at $O y$-axis. This approach allows us to solve the entire solution in the twodimensional case and implement the corresponding software algorithm.

\section{CONCLUSIONS}

Since the mathematical modelling using colloidal adsorbents to the purification processes is new, the importance of new mathematical models become obvious. The statement and the mathematical modelling of the new corresponding twodimensional problem of contaminant migration in unsaturated porous media was formulated. The numerical solution of the boundary value problem was found by the finite difference method using locally one-dimensional Samarsky's method and monotonic difference schemes and a computation algorithm is proposed as well. The results might be used to build effective risk assessment strategies for cleaning and further useful use of the soil massifs.

\section{ACKNOWLEDGMENT}

This research work was conducted as part of the young scientist research project \#0120U000235 and funded by the national budget of Ukraine.

\section{REFERENCES}

[1] N. Remez, T. Osipova, O. Kraychuk, and S. Kraychuk, "Simulation of the solid waste landfill settlement taking into account underlying soil," EEJET, vol. 3, 10(81), p. 12, 2016, doi: 10.15587/1729-4061.2016.72333.

[2] A. Pligovka, P. Yunin, A. Hoha, S. Korolyov, G. Gorokh, and E. Skorokhodov, "Morphology and Structure of Defected Niobium Oxide Nonuniform Arrays Formed by Anodizing Bilayer Al/Nb Systems," Technical Physics, vol. 90, no. 11, p. 1854, 2020, doi: 10.1134/S1063784220110213.

[3] A. Poznyak, A. Pligovka, U. Turavets, and M. Norek, "On-Aluminum and Barrier Anodic Oxide: Meeting the Challenges of Chemical Dissolution Rate in Various Acids and Solutions," Coatings, vol. 10, no. 9, p. 875, 2020, doi: 10.3390/coatings10090875.

[4] M. I. Romashchenko, V. O. Bohaienko, T. V. Matiash, V. P. Kovalchuk, and I. I. Danylenko, "Influence of evapotranspiration assessment on the accuracy of moisture transport modeling under the conditions of sprinkling irrigation in the south of Ukraine," Archives of Agronomy and Soil Science, vol. 1, no. 2, pp. 1-12, 2019, doi: 10.1080/03650340.2019.1674445.

[5] A. Rokochinskiy, P. Volk, O. Pinchuk, V. Turcheniuk, N. Frolenkova, and I. Gerasimov, "Forecasted estimation of the efficiency of agricultural drainage on drained lands," Journal of Water and Land Development, vol. 40, no. 1, pp. 149-153, 2019, doi: 10.2478/jwld-2019-0016.
[6] A. Safonyk, O. Prysiazhniuk, and I. Prysiazhniuk, "Modeling of the Processes of Heat and Mass Transfer in the Thin Tube Given the Conditions of Exchange with Surrounding Soil," in 2019 IEEE 14th International Conference on Computer Sciences and Information Technologies (CSIT), 2019, pp. 100-103.

[7] A. P. Vlasyuk and V. V. Zhukovskii, "Mathematical Simulation of the Migration of Radionuclides in a Soil Medium Under Nonisothermal Conditions with Account for Catalytic Microparticles and Nonlinear Processes," Journal of Engineering Physics and Thermophysics, vol. 90, no. 6, pp. 1386-1398, 2017, doi: 10.1007/s10891-017-1697-4.

[8] A. P. Vlasyuk, R. V. Kochan, V. V. Zhukovskyy, and N. A. Zhukovska, "Mathematical and computer modeling of contaminant migration to filter trap in two-dimensional nonlinear case," 18th International Multidisciplinary Scientific Geoconference SGEM 2018, vol. 18, 2.2, pp. 293-300, 2018, doi: 10.5593/sgem2018/2.2/S08.037.

[9] M. Auffan et al., "Nanomaterials as adsobents," (in af), Environmental Nanotechnology: Applications and Impacts of Nanomaterials, McGraw-Hill, New York, pp. 371-392, 2007.

[10] P. P. Kostrobij, O. V. Viznovych, B. B. Markiv, and M. V. Tokarchuk, "Generalized kinetic equations for dense gases and liquids in the Zubarev nonequilibrium statistical operator method and Renyi statistics," Theor Math Phys, vol. 184, no. 1, pp. 1020-1032, 2015, doi: 10.1007/s11232-015-0314y.

[11] D. Atkinson and C. A. Watson, Eds., The science beneath organic production. Hoboken NJ: Wiley Blackwell, 2020.

[12] O. Y. Chernukha, "Physico-mathematical models of heterodiffusion in a layer," J Math Sci, vol. 86, no. 2, pp. 2573-2577, 1997, doi: 10.1007/BF02356099.

[13] J. Kärger and D. M. Ruthven, "Diffusion in nanoporous materials: fundamental principles, insights and challenges," New J. Chem., vol. 40, no. 5, pp. 4027-4048, 2016, doi: 10.1039/C5NJ02836A.

[14] B. M. Budak, A. A. Samarskii, A. N. Tikhonov, I. N. Sneddon, M. Stark, and S. Ulam, A Collection of Problems on Mathematical Physics: International Series of Monographs in Pure and Applied Mathematics: Elsevier Science, 2013.

[15] I. V. Sergienko, V. V. Skopetskii, and V. S. Deineka, Mathematical Simulation and Investigation of Processes in Inhomogeneous Media. Kiev: Naukova Dumka, 1991.

[16] A. S. Kolesnikov, I. V. Sergeeva, N. E. Botabaev, A. Z. Al'zhanova, and K. A. Ashirbaev, "Chemical and phase transitions in oxidized manganese ore in the presence of carbon," Steel Transl., vol. 47, no. 9, pp. 605-609, 2017, doi: 10.3103/S0967091217090078.

[17] I. K. Gavich, Hydrogeodynamics. Rotterdam: Balkema, 1997.

[18] O. A. Ladyzenskaja, V. A. Solonnikov, N. N. Ural'ceva, and S. Smith, Linear and quasi-linear equations of parabolic type, 5th ed. Providence, RI: American Mathematical Soc, 1998.

[19] A. A. Samarskii, The theory of difference schemes. New York: Marcel Dekker, 2001.

[20] S. V. Fedorov, "On the selection of a step by time, when solving nonstationary heat conductivity problems with the method of finite elements," AM\&FI, vol. 5, no. 1, pp. 21-30, 2018, doi: 10.25206/2311-49082018-5-1-21-30.

[21] S. V. Fedorov, "Decrease in errors at solution of problems of heat conductivity by finite element method," OSB, no. 160, pp. 169-173, 2018, doi: 10.25206/1813-8225-2018-160-169-173.

[22] A. Vlasyuk, V. Zhukovskyy, N. Zhukovska, O. Pinchuk, and H. Rajab, "Mathematical Modeling of Heat, Mass and Moisture Transfer in Catalytic Porous Media," WSEAS Transactions on Applied and Theoretical Mechanic, vol. 15, pp. 52-59, 2020, doi: 10.37394/232011.2020.15.8. 